\title{
Percepção dos profissionais de saúde de um hospital público em relação à ortotanásia
} em pacientes idosos

\author{
Perception of health professionals of one public hospital in relation to ortotanasia in elderly \\ patients
}

Percepción de los profesionales de la salud de un hospital público en relación con la ortotanasia en pacientes de edad avanzada

Recebido: 31/12/2020 | Revisado: 02/01/2021 | Aceito: 05/01/2021 | Publicado: 07/01/2021

Thayse Sousa de Vasconcelos ORCID: https://orcid.org/0000-0001-6625-6437 Centro Universitário UNIFACISA, Brasil E-mail: thaysevasconcelosfisio@gmail.com

Ana Gonçalves Lima Neta ORCID: https://orcid.org/0000-0001-6684-1900 Centro Universitário UNIFACISA, Brasil E-mail: anagoncalves.noronha@gmail.com

Tatiane Carvalho Brandão ORCID: https://orcid.org/0000-0002-9273-2895 Centro Universitário UNIFACISA, Brasil

E-mail: tati.carvalho13@gmail.com

Kedma Anne Lima Gomes ORCID: https://orcid.org/0000-0001-6720-011X Centro Universitário UNIFACISA, Brasil

E-mail: kedmaannekel@gmail.com

Jéssica Leite

ORCID: https://orcid.org/0000-0002-4726-9416 Centro Universitário UNIFACISA, Brasil

E-mail: jessica.leite@maisunifacisa.com.br

\begin{abstract}
Resumo
Objetivos: Investigar a percepção dos profissionais que atuam em um hospital público, com relação à ortotanásia e cuidados paliativos em idosos, considerando também o perfil dos profissionais de saúde que atuam no local, se o tipo de formação profissional repercute sobre as escolhas da equipe frente à terminalidade da vida e a necessidade de atualização dos profissionais sobre a ortotanásia. Métodos: Tratou-se de uma pesquisa transversal, com abordagem quali-quantitativa, em um hospital público de Campina Grande-PB, com aplicação de entrevista a uma equipe multiprofissional. Resultados: Percebeu-se que a ortotanásia é conhecida pelos profissionais, embora poucos sintam-se aptos a realizá-la. Notou-se pouco conhecimento destes para instituir cuidados paliativos, fenômeno que não mostrou associação significativa com o tipo de formação (Qui-quadrado $=0.98, \mathrm{p}=0.32$ ) ou o tipo de instituição em que concluiu a formação (Qui-quadrado=1.63, $\mathrm{p}=0.20$ ), mas apresentou relação moderada ( $\mathrm{V}$ de Cramer $=0.48, \mathrm{p}=0.002$ ) e significativa (Qui-quadrado=9.40, $\mathrm{p}=0.002$ ) com o fato de o profissional possuir ou não alguma especialização. Sobre as dificuldades enfrentadas nos cuidados com os pacientes terminais, lidar com a família destes foi considerada a principal. Quanto às ferramentas necessárias para viabilizar a ortotanásia em idosos, melhorar o conhecimento e a abordagem sobre o tema teve maior prevalência. Conclusão: Concluiu-se que há necessidade da ampliação da temática na formação dos profissionais, a fim de possibilitar uma assistência verdadeiramente humanizada ao idoso.
\end{abstract}

Palavras-chave: Idosos; Hospitalização; Profissionais de saúde; Ortotanásia.

\begin{abstract}
Objectives: To investigate the perception of professionals who work in a public hospital, in relation to orthothanasia and palliative care in the elderly, also considering the profile of health professionals working in the place, if the type of professional training affects the team's choices to the end of life and the need to update professionals on orthothanasia. Methods: This was a cross-sectional study, with a qualitative and quantitative approach, in a public hospital in Campina Grande-PB, with the application of an interview to a multidisciplinary team. Results: It was noticed that orthothanasia is known by professionals, although few feel able to perform it. There was little knowledge of these to institute palliative care, a phenomenon that did not show a significant association with the type of training $($ Chi-square $=0.98, p=0.32$ ) or the type of institution where the training was completed (Chi-square $=1.63, \mathrm{p}=0.20$ ), but presented a moderate (Cramer $\mathrm{V}=0.48, \mathrm{p}=0.002$ ) and significant (Chi-square $=9.40, \mathrm{p}=0.002$ ) relationship with the fact that the
\end{abstract}


professional has or not some specialization. Regarding the difficulties faced in caring for terminally ill patients, dealing with their families was considered the main one. As for the tools necessary to make orthothanasia feasible in the elderly, improving knowledge and approach on the topic had a higher prevalence. Conclusion: It was concluded that there is a need to expand the theme in the training of professionals, in order to enable truly humanized assistance to the elderly.

Keywords: Elderly; Hospitalization; Health professionals; Orthothanasia.

\section{Resumen}

Objetivos: Investigar la percepción de los profesionales que laboran en un hospital público, en relación a la ortotanasia y los cuidados paliativos en personas mayores, considerando también el perfil de los profesionales de la salud que actuan en el lugar, si el tipo de formación profesional afecta las elecciones del equipo al final de la vida y la necesidad de actualizar a los profesionales sobre la ortotanasia. Métodos: Se trata de un estudio transversal, con abordaje cualicuantitativa, en un hospital público de Campina Grande-PB, con la aplicación de una entrevista a un equipo multidisciplinario. Resultados: Se notó que la ortotanasia es conocida por los profesionales, aunque pocos se sienten capaces de realizarla. Se tenía poco conocimiento para instituir cuidados paliativos, fenómeno que no mostró asociación significativa con lo entrenamiento (Chi-cuadrado $=0.98, \mathrm{p}=0.32$ ) o la institución donde se completó el entrenamiento (Chi-cuadrado $=1,63, \mathrm{p}=0,20)$, pero presentó una relación moderada ( $\mathrm{V}$ de Cramer $=0,48, \mathrm{p}=0,002)$ y significativa (Chi-cuadrado $=9,40, \mathrm{p}=0,002$ ) con que el profesional tenga o no especialización. En cuanto a las dificultades con el cuidado de los enfermos terminales, el trato con sus familiares fue el principal. En cuanto a las herramientas necesarias para hacer factible la ortotanasia a las personas mayores, la mejora del conocimiento y abordaje del tema tuvo una mayor prevalencia. Conclusión: Se concluyó que existe la necesidad de ampliar en la formación de profesionales, a fin de posibilitar una asistencia humanizada a las personas mayores.

Palabras clave: Personas mayores; Hospitalización; Profesionales de la salud; Ortotanasia.

\section{Introdução}

De acordo com Souza et al. (2020), A população idosa vem crescendo ao longo das últimas décadas. Nos afirma também, que tal fenômeno vem acompanhado pelo acréscimo da prevalência de Doenças Crônicas Não Transmissíveis (DCNTs). Uma grande parcela de idosos sofre as consequências de DCNTs não controladas e ficam predispostos ao desenvolvimento de limitações funcionais e incapacidades permanentes, associadas ao aumento dos índices de hospitalização e mortalidade (OPAS, 2016).

A hospitalização é considerada um processo de grande risco principalmente para essa população, pois devido a diminuição na capacidade funcional de atividades fisiológicas, o idoso fica mais propenso a complicações causadas pelo repouso prolongado no leito durante a hospitalização que podem comprometer a independência, autonomia e qualidade de vida (Silva et al., 2020)

Em hospitalizações prolongadas que cursam com a morte, a equipe de saúde fica mais próxima ao paciente e sua família, o que pode causar sentimentos de ineficácia, frustração e culpabilidade, que podem interferir na prestação do cuidado. Portanto, há a necessidade de expansão de discussões que envolvam aspectos relativos à morte, a fim de minimizar o sofrimento pessoal dos profissionais e qualificar a assistência (Both et al., 2013).

Nesse contexto, tem sido levantado o conceito de morte digna ou ortotanásia. Porém, essa definição nem sempre é a mesma para os pacientes, familiares e profissionais de Saúde. A abreviação da morte, a aplicação de esforços terapêuticos desproporcionais, como a oposição, futilidade ou instituição dos cuidados paliativos, que suavizam o sofrimento, compõem os fins de tratamentos que podem ser oferecidos ao indivíduo em estágio terminal (Felix et al., 2013).

De acordo com Carvalho et al. (2015), os cuidados paliativos devem ser oferecidos a indivíduos em estado terminal, de modo a garantir uma abordagem que melhore a qualidade de vida desses e de seus familiares, mediante prevenção e alívio de sofrimento, pela observação precoce e tratamento da dor ou de outros problemas físicos, psicológicos, sociais e espirituais, estendendo-se, inclusive, à fase de luto.

Segundo Almeida e Freitas (2018), um paciente sob cuidados paliativos é aquele cujos recursos conhecidos para a cura da doença esgotaram-se. O direito de morrer com dignidade significa que as pessoas podem viver os últimos dias rodeadas de amor e amparo. A ortotanásia permite que o paciente em fase terminal enfrente o processo da morte de forma mais tranquila, o 
objetivo não é mais curar e sim manter a vida enquanto for favorável ao paciente, permitindo que a morte chegue naturalmente (Cano et al., 2020).

Uma das dificuldades para utilização dos cuidados paliativos no Brasil é a carência de sua abordagem no ensino educacional de profissionais de saúde, o que reflete no desempenho destes (Carvalho et al., 2017). O despreparo dos profissionais para lidar com a morte tem como uma das suas causas, o ensino nos cursos da área da saúde que enfatizam a formação técnicocientífica, propiciando pouco espaço para a abordagem dos aspectos emocionais, espirituais e sociais. Nesse sentido, Bifulco e Lochida (2009), nos afirmam que muitas vezes a morte pode ser relacionada com derrota, perda e frustração, o oposto da meta dos cursos da área da saúde, que é a cura.

Para realização da pesquisa levou-se em consideração o fato de que a ortotanásia é a morte no tempo certo, sem uso de condutas terapêuticas que prolonguem o sofrimento. Partiu-se do pressuposto de que para que a equipe de saúde aceite essa prática é necessário que haja compreensão clara das questões legais envolvidas, bem como entendimento do processo natural e cronológico da vida, que envolve a morte, entretanto, essa compreensão não é simples, pois a morte coloca o profissional de saúde diante de sua própria vida, gerando conflitos e dúvidas sobre a eficácia, objetivos e relevância de seus cuidados.

Diante do exposto, esta pesquisa teve como objetivo geral investigar o entendimento dos profissionais que atuam em um hospital público, no que diz respeito à ortotanásia e cuidados paliativos em pacientes idosos, como objetivos específicos buscou-se traçar o perfil dos profissionais de saúde que atuam no local; verificar se o tipo de formação profissional repercute sobre as escolhas terapêuticas da equipe frente à terminalidade da vida e verificar a necessidade de atualização dos profissionais nos conceitos de ortotanásia e cuidados paliativos em geriatria.

\section{Métodos}

Trata-se de uma pesquisa do tipo transversal, com abordagem quali-quantitativa, realizada em um hospital público de Campina Grande-PB, a qual é conceituada por Creswell (2010), como uma abordagem em que correlaciona os pontos principais tanto da quantitativa quanto da qualitativa, favorecendo a pesquisa. A amostra foi do tipo não-probabilística, de base institucional, formada por 40 profissionais que atuavam regularmente com a população geriátrica do Hospital. Utilizou-se como parâmetro da saturação amostral, o esgotamento de novos assuntos no discurso dos respondentes (Flick, 2009). Foram excluídos os profissionais que se recusaram a participar da pesquisa e os estagiários que estavam realizando visitas observacionais ou estágios de curta duração.

A coleta de dados foi realizada após aprovação pelo Comitê de Ética em Pesquisa do CESED, parecer: 80727617.2.0000.5175, todos os indivíduos participantes assinaram o TCLE atendendo à Resolução CNS 466/12, através de um roteiro de entrevista semiestruturado e categorizado elaborado pelos autores, contendo características sócio-demográficas e profissionais da amostra, como também questões referentes a percepção dos cuidados paliativos, conceitos de morte digna e a experiência desse profissional em relação a aplicação desses conceitos na prática. As entrevistas foram realizadas no próprio hospital em sala reservada e sem interrupções.

Para a análise quantitativa foi utilizado o Pacote estatístico SPSS ${ }^{\circledR}$ versão 20.0, os dados foram descritos em média, desvio padrão ( $( \pm)$, intervalo de confiança (IC95\%) e distribuição de frequências, para estabelecer a existência de associação entre as variáveis qualitativas foi utilizado o teste Qui-quadrado $\left(\mathrm{X}^{2}\right)$ e o $\mathrm{V}$ de Cramer para medir a força dessa associação, sendo considerada uma relação significativa quando $\mathrm{p}<0,05$. Já na análise qualitativa, foi utilizada como técnica exploratória a análise de conteúdo temática, foi realizada a leitura do material transcrito, o que permitiu organizar aspectos importantes para a análise, em seguida o material foi explorado e agrupado em categorias pré-estabelecidas e por fim, os dados foram interpretados, o que permitiu fazer as inferências sobre o conteúdo obtido (Bardin, 2011). Para identificação dos entrevistados foi atribuída a letra inicial da profissão e a ordem em que foi entrevistado, seguida da idade. 


\section{Resultados e Discussão}

No presente estudo, foram entrevistados 40 profissionais de saúde do Hospital, dentre eles: 8 fisioterapeutas, 9 enfermeiros, 5 médicos, 2 nutricionistas, 2 assistentes sociais e 14 técnicos de enfermagem.

No que diz respeito ao perfil sócio-demográfico dos profissionais participantes da pesquisa (Tabela 1), verificou-se que a maior parte da amostra foi composta por técnicos de enfermagem, que somados a enfermeiros ultrapassaram a metade da amostra, fato semelhante foi encontrado por Silva, Resende e Andrade (2019) em seu estudo sobre a atitude dos profissionais de saúde diante da morte, no qual verificou-se que os profissionais da área de enfermagem foram os mais predominantes. A média de idade foi de 37.2 anos com desvio padrão de 9.24 anos.

Tabela 1 - Caracterização da amostra.

\begin{tabular}{|c|c|c|}
\hline Características & $\mathbf{N}^{\mathbf{0}}$ & $\%$ \\
\hline \multicolumn{3}{|l|}{ Formação profissional } \\
\hline Médico & 05 & $12,5 \%$ \\
\hline Fisioterapeuta & 08 & $20 \%$ \\
\hline Nutricionista & 02 & $5 \%$ \\
\hline Assistente social & 02 & $5 \%$ \\
\hline Enfermeiro & 09 & $22,5 \%$ \\
\hline Técnico de enfermagem & 14 & $35 \%$ \\
\hline \multicolumn{3}{|l|}{ Ano de conclusão } \\
\hline Menos de 05 anos & 12 & $30 \%$ \\
\hline Mais de 05 anos & 14 & $35 \%$ \\
\hline Mais de 10 anos & 14 & $35 \%$ \\
\hline \multicolumn{3}{|l|}{ Instituiçãa } \\
\hline Pública & 17 & $42,5 \%$ \\
\hline Privada & 23 & $57,5 \%$ \\
\hline \multicolumn{3}{|l|}{ Especialização } \\
\hline Sim & 24 & $60 \%$ \\
\hline Não & 16 & $40 \%$ \\
\hline \multicolumn{3}{|l|}{ Mestrado } \\
\hline Sim & 05 & $12,5 \%$ \\
\hline Não & 35 & $87,5 \%$ \\
\hline \multicolumn{3}{|l|}{ Doutorado } \\
\hline Não & 40 & $100 \%$ \\
\hline \multicolumn{3}{|c|}{ Tempo de atuação profissional } \\
\hline Menos de 05 anos & 11 & $27,5 \%$ \\
\hline Mais de 05 anos & 13 & $32,5 \%$ \\
\hline Mais de 10 anos & 16 & $40 \%$ \\
\hline \multicolumn{3}{|l|}{ Atuação na instituição } \\
\hline Menos de 05 anos & 31 & $77,5 \%$ \\
\hline Mais de 05 anos & 04 & $10,0 \%$ \\
\hline Mais de 10 anos & 05 & $12,5 \%$ \\
\hline \multicolumn{3}{|c|}{ Formação complementar em Geriatria e Gerontologia } \\
\hline Sim & 01 & $2,5 \%$ \\
\hline Não & 39 & $97,5 \%$ \\
\hline
\end{tabular}


Fonte: Dados da Pesquisa, Brasil (2020).

A Tabela 1 apresenta as características da amostra estudada quanto a formação e atuação profissional, e suas respectivas porcentagens.

Sobre o tempo de formação, 35\% concluiu sua graduação há mais de 10 anos, sendo 57,5\% em instituições privadas. Além disso, $60 \%$ destes profissionais possuem especialização, 12,5\% possui mestrado e nenhum cursou doutorado. Ainda sobre o preparo acadêmico da amostra, destaca-se que $97,5 \%$ não possui nenhuma formação complementar em geriatria e gerontologia. Estes achados seguem de forma semelhante ao estudo de Moritz e Nassar (2004), pois a maior parte dos participantes dessa pesquisa também concluíram sua graduação em instituições privadas e iniciaram uma especialização para aperfeiçoamento profissional, ao passo que uma pequena parte iniciou mestrado e nenhuma chegou ao doutorado.

Diante do exposto, a caracterização dos participantes do estudo da amostra corrobora algumas informações referentes ao desenvolvido por Silva et al. (2016), no qual foram entrevistados profissionais de um hospital sobre os conceitos distanásia, eutanásia e ortotanásia e possíveis implicações bioéticas no cuidado do doente terminal, levando em consideração a especialização dos profissionais, tempo de formação e tempo de atuação na UTI.

Gráfico 1 - Conhecimento dos profissionais de saúde acerca da ortotanásia e cuidados paliativos.

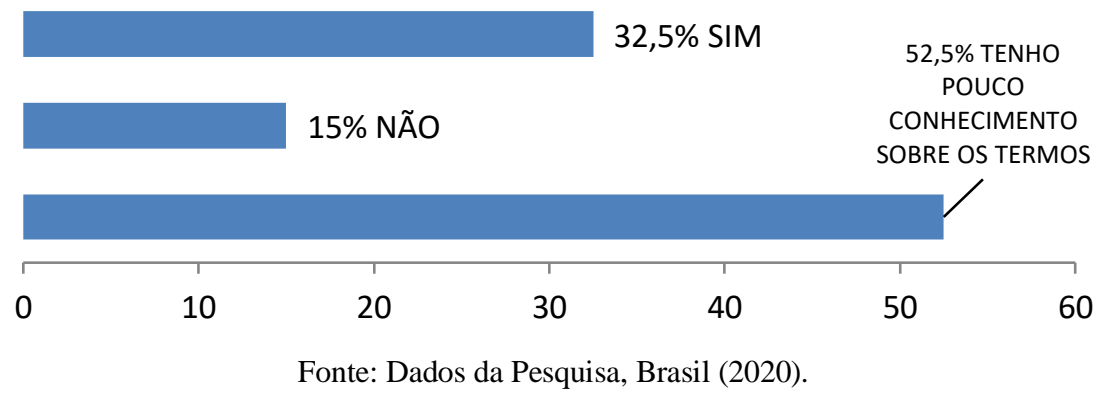

O Gráfico 1, acima, reflete o quanto os profissionais pesquisados julgam ter conhecimento sobre o tema ortotanásia, de modo que mais da metade da amostra refere conhecer o tema. Porém, quando questionados sobre o preparo da equipe para realização da ortotanásia nos pacientes idosos, apenas $8 \%$ dos entrevistados consideraram que toda a equipe se encontrava preparada para realização, conforme demonstrado no Gráfico 2, a seguir.

Gráfico 2 - Percepção dos profissionais quanto à equipe sentir-se preparada para realização da ortotanásia.

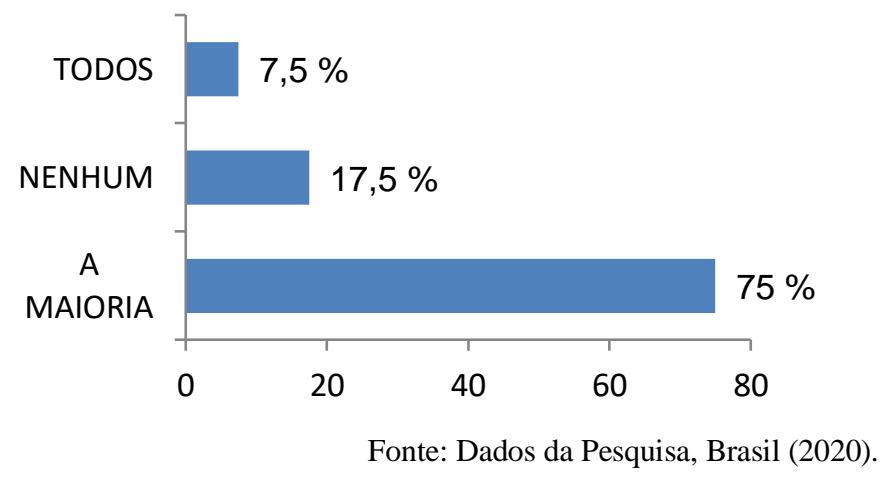

Em pesquisa similar, Meneguim e Ribeiro (2016) em concordância com o presente estudo, apontam que a falta de conhecimento do profissional de saúde sobre como agir em situações relacionadas à morte estabelece empecilhos para estes realizarem os cuidados paliativos, e dificulta o atendimento do profissional ao paciente. Esse fato se torna ainda mais importante quando se leva em consideração o aumento do envelhecimento populacional, como também a crescente prevalência de doenças 
crônicas não transmissíveis, suscitando a necessidade de cuidados no fim da vida e exigindo uma assistência especial aos pacientes em fase terminal.

Em consonância com os resultados da presente pesquisa, Oneti, Barreto e Martins (2017) descrevem em seu estudo sobre percepção dos profissionais de enfermagem frente à prática da distanásia e ortotanásia que a falta de conhecimento sobre estes temas citados, acaba levando os profissionais a se questionarem sobre o momento em que a equipe deve investir em recursos para atender determinado paciente, ou qual a melhor decisão a tomar, mesmo que possa levar o doente a um sofrimento prolongado.

No que se refere ao conhecimento sobre qual situação está indicado o tratamento paliativo no idoso, verificou-se que $20 \%$ da amostra afirmou não saber. Esse fato, pode ser explicado por Santana et al. (2014) em seu estudo sobre a visão multidisciplinar acerca do morrer com dignidade, onde descrevem que a aceitação em relação a morte para os profissionais que a assiste, causa certa fragilidade emocional para estes, quando necessitam acompanhar de perto a morte de seu paciente.

Ainda de acordo com os mesmos autores, nota-se o pouco conhecimento dos profissionais de saúde em relação a qual situação utilizar os cuidados paliativos, mesmo observando uma preocupação destes profissionais em melhorar a condição de vida do doente na sua fase terminal.

Para verificar se o tipo de formação profissional repercute sobre as escolhas terapêuticas da equipe frente à terminalidade da vida foi realizado um teste de associação entre algumas perguntas do questionário e informações acerca do tipo de formação de cada profissional, se este possuía formação superior ou técnica, se havia concluído seu curso em instituição pública ou privada e se possuía especialização em qualquer área.

Em relação ao conhecimento desses profissionais sobre qual situação é indicado o tratamento paliativo em pacientes idosos, não foi encontrada relação significativa com o tipo de formação (Qui-quadrado= $0.98, p=0.32$ ) e tipo de instituição (Qui-quadrado $=1.63, \mathrm{p}=0.20$ ), porém a relação se mostrou moderada $(\mathrm{V}$ de Cramer $=0.48, \mathrm{p}=0.002)$ e significativa (Quiquadrado $=9.40, \mathrm{p}=0.002$ ) com o fato do profissional possuir ou não especialização em alguma área. De todos os profissionais que possuem especialização (24 participantes), 95,8\% (23 participantes) consideram saber em que situação indicar e 4,2\% (1 participantes) não considera, e de todos os profissionais sem especialização (16 participantes), 56,3\% (9 participantes) consideram saber em que situação indicar e 43,8\% (7 participantes) não considera.

Já quando questionados se acreditavam que todas as medidas terapêuticas possíveis para prolongar a vida de um paciente idoso em fase terminal deveriam ser aplicadas, não houve relação significativa com nenhuma das variáveis referentes a formação.

Levando em consideração os aspectos para a realização do cuidado paliativo, Cardoso et al. (2013) descrevem em seu estudo sobre a vivência da equipe multiprofissional na assistência hospitalar, que quanto mais qualificado o profissional for, mais condições terão de proporcionar um atendimento de uma forma harmoniosa para seu paciente. Corroborando neste sentido, que os profissionais especializados e capacitados para atenderem pacientes na fase terminal de suas vidas, demonstram melhores resultados no modo do cuidado dos sintomas destes, sendo estes sintomas emocionais ou físicos.

Portanto, Guimarães e Nunes (2016) relatam em seu estudo sobre conhecimento do profissional de enfermagem sobre cuidados paliativos em pacientes oncológicos, que os profissionais da saúde em geral necessitam aumentar seus conhecimentos no que diz respeito ao cuidado dos pacientes na fase terminal de suas vidas, pois estes profissionais devem estar aptos a proporcionar e melhorar a qualidade de vida destes pacientes, como também buscar um melhor entendimento em relação aos entraves que a doença e a morte podem ocasionar.

Já em relação as emoções vivenciadas pelos profissionais durante o processo de morte de um paciente, quando questionado se o profissional se considerava preparado para aceitar a morte de um paciente idoso e lidar com a fase de luto dos familiares, evidenciou-se para este fato, que a maioria $(80 \%)$ se acha preparado para essa aceitação. Porém, mesmo julgando-se 
preparados para lidar e aceitar esse momento o Gráfico 3 demonstra que $62 \%$ dos profissionais sentem-se angustiados ao falar sobre a morte de seus pacientes.

Gráfico 3 - Presença de sentimento de angústia do profissional de saúde ao falar sobre a morte do paciente.

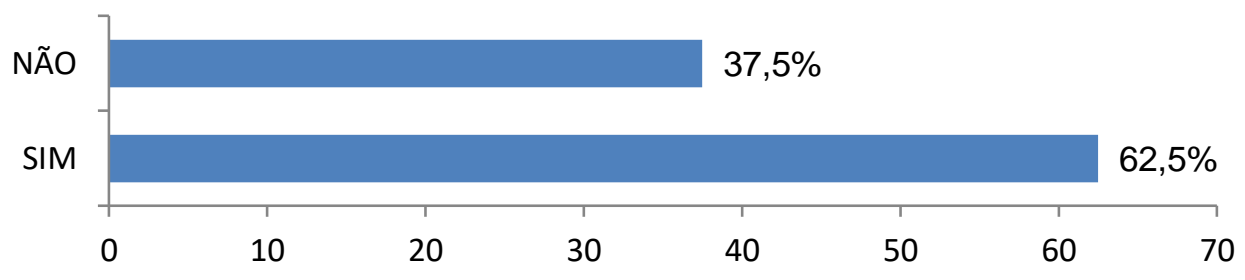

Fonte: Dados da Pesquisa, Brasil (2020).

Sobre esse fato, Mendes, Lustosa e Andrade (2009) relatam que as equipes de saúde na maioria das vezes encontramse despreparadas para lidar com a morte de um paciente, pois esse momento representa uma sensação de fracasso, angústia e não aceitação em relação a não cumprir uma missão que lhe foi dada. Já Giaretton (2013), refere que a maioria dos profissionais da saúde se opõem em vivenciar o luto de um paciente de uma maneira completa, manifestando impotência diante da morte, pelo fato de não poder curar o paciente.

Desta maneira, confirmando o que aponta a literatura, ao questionar os profissionais sobre as emoções que estes vivenciavam quando medidas terapêuticas eram aplicadas para prolongar a vida de um paciente idoso em fase terminal, 35,\% referiram procurar não se envolver emocionalmente nesse processo, enquanto 32,5\% disseram sentir angústia, frustração e raiva, por outro lado, 25\% mencionaram aceitação, alívio e sensação de dever cumprido, e por fim 7,5\% mencionaram que não conseguiam lembrar das emoções no momento. De forma estabelecida, no curso da hospitalização de um enfermo, vários profissionais presenciam o processo de morte, e estes de um jeito ou de outro acabam lidando com grandes emoções, ficando então frente a frente com suas próprias limitações, o que consequentemente gera angústias e aflições (Santos, Menezes e Gradvohl, 2013).

Santana et al. (2014), descrevem que independente dos sentimentos que envolvem o processo de morte de um paciente, torna-se significante o profissional de saúde ter o comprometimento de oferecer uma morte digna para esse, pois cuidar de enfermos em fase terminal requer um cuidado mais expressivo na valorização da vida do ser humano.

No presente estudo, todos os participantes acham de fundamental importância ampliar a discussão sobre a ortotanásia nos cursos de graduação, tornando-se clara a necessidade da abordagem dessa temática na vivência prática desses profissionais. Quando questionados se já haviam discutido sobre a temática em seu ambiente de trabalho, $75 \%$ desses profissionais responderam que não.

No que tange aos questionamentos discursivos, os profissionais responderam perguntas sobre as dificuldades que vêm enfrentado no cuidado aos pacientes idosos em fase terminal e o que seria necessário para viabilizar a realização da ortotanásia

Após as respostas passarem pela Análise de Conteúdo temática, as narrativas da amostra possibilitaram a construção das categorias e subcategorias registradas no Quadro Analítico (Quadro 1). 
Quadro 1. Quadro analítico com as categorias e subcategorias.

\begin{tabular}{|c|c|}
\hline CATEGORIAS & SUBCATEGORIAS \\
\hline \multirow{2}{*}{ Falta de preparo diante da situação } & Aceitação familiar \\
\cline { 2 - 2 } & Qualificação limitada do profissional \\
\hline \multirow{2}{*}{ Alternativas para possibilitar a realização da ortotanásia } & Capacitação profissional \\
\cline { 2 - 2 } & Suporte aos familiares \\
\hline
\end{tabular}

Fonte: Dados da Pesquisa, Brasil (2020).

O Quadro 1 apresenta a construção analítica com a descrição das categorias temáticas e subcategorias que serão detalhados a seguir.

\section{Falta de preparo diante da situação}

No que diz respeito às dificuldades que esses profissionais têm enfrentado no cuidado aos pacientes idosos em fase terminal, percebe-se que a aceitação dos familiares é o tópico mais mencionado:

\footnotetext{
"A família muitas vezes desconhece o assunto e não aceita" (E-1, 38 anos).

"A maior dificuldade é a aceitação da família" (TE-39, 35 anos)

"Dificuldade em relação ao sofrimento do mesmo e de seus familiares" (F-32, 28 anos).
}

Sobre isso, Mendes, Lustosa e Andrade (2009) descrevem em seu estudo sobre paciente terminal, família e equipe de saúde, que além dos pacientes, os profissionais de saúde necessitam ter um determinado cuidado também com os familiares destes, pois apesar da dificuldade relatada pela maioria dos profissionais em lidar com a família, esse momento faz parte do processo de cuidado em saúde, e deve ser feito de uma forma igualmente humanizada, pois estes familiares também são afetados emocionalmente ao saber que um ente querido encontra-se em situação de fase terminal da vida.

O período próximo à morte de um familiar ou pessoa querida gera reflexões e mudanças na estrutura psicológica, afetiva, social e física de uma família ou de um grupo social, o que reforça a necessidade de analisar minuciosamente os medos, receios e dúvidas das pessoas que possuíram ou possuem familiares e amigos com algum agravo à saúde, levando em consideração questões culturais, sociais e até demográficas (Santana, Rigueira e Dutra, 2010).

Adicionalmente, é possível identificar nas falas que a qualificação dos profissionais para lidar com a ortotanásia ainda ocorre de maneira limitada:

"O pouco conhecimento dos profissionais relacionados aos cuidados paliativos, terminalidade da vida, distanásia, eutanásia, ortotanásia...os leva a condutas inapropriadas e muitas vezes indignas para aquele paciente que vivencia sua fase terminal" (E-25, 26 anos)

\footnotetext{
“A falta de formação complementar para os profissionais da saúde” (N-7, 34 anos)

"Conhecimento do tema ortotanásia sobre os profissionais de saúde” (M-27, 34 anos)
}

"Falta de conhecimento dos profissionais" (E-38, 39 anos) 
Um estudo realizado no Rio Grande do Sul, cujo objetivo foi verificar a percepção do enfermeiro em relação à ortotanásia, constatou igualmente que profissionais da saúde recebem pouca informação com relação à morte durante sua formação acadêmica e que, mesmo sendo um termo pouco conhecido, é uma circunstância que ocorre na rotina hospitalar, devendo o profissional atuar como um elo de ligação entre paciente, família e equipe (Balla e Haas, 2008). Nesse sentido, surge a necessidade de reformulação das matrizes curriculares dos cursos de saúde para que possam ser inclusos os cuidados paliativos e a necessidade de ampliar a discussão sobre a temática (Arrieira et al., 2018).

Conforme percepção da Academia Nacional de Cuidados Paliativos (ANCP), o ensino sobre esses cuidados nas graduações da área da saúde não está especificamente contemplado pelo Ministério da Educação (MEC), não havendo uma regulamentação apontando sobre uma matriz de competências que direcione cursos e residências (Forte, 2018).

\section{Alternativas para possibilitar a realização da ortotanásia}

$\mathrm{Na}$ questão referente ao que é necessário para viabilizar a realização da ortotanásia em pacientes idosos na fase terminal da vida, verificou-se que a falta de capacitação e de conhecimento sobre o tema foi a resposta mais recorrente, reforçando o que foi descrito anteriormente, como também demonstrado nas falas de alguns participantes:

"Para melhorar a realização da ortotanásia, seria importante um momento de capacitação para todos os profissionais que de forma direta ou indireta tem acesso a esses pacientes, pois acredito que uma grande maioria sinta dificuldades em tal procedimento" (TE-04, 27 anos)

"Melhorar conhecimento do tema pelos profissionais de saúde" (M-27, 34 anos)

"É necessário um trabalho de humanização dos profissionais de saúde. Promover cursos, palestras, e discussões sobre a ortotanásia através de capacitações dos profissionais” (F-36, 50 anos)

De acordo com o exposto, Borges e Mendes (2012) relatam que durante a formação acadêmica, o profissional de saúde não adquire os conhecimentos necessários e suficientes para entender a morte e o processo de morrer. A morte é uma temática pouco abordada e discutida na formação acadêmica. Embora seja um acontecimento natural que está presente na vida das pessoas e desses profissionais, a morte é de difícil aceitação, além de ser pouco discutida no dia a dia dos indivíduos que lidam diretamente com ela (Nogueira, Oliveira e Pimentel, 2006).

E por fim, Silva et al. (2014) expuseram em estudo sobre o comportamento dos profissionais de saúde em relação a morte dos pacientes, a necessidade de aumentar a abordagem sobre a temática morte e fim da vida. Pois se observou que de acordo com a cultura ocidental, o tema morte é na maioria das vezes evitado, pois trata-se de um contexto associado a perda, a impotência e sentimento de dor.

\section{Conclusão}

No que diz respeito a percepção dos profissionais em relação à cuidados paliativos e ortotanásia em pacientes idosos, foi possível identificar no presente estudo que a maioria dos profissionais sabe em qual situação seria indicado o tratamento paliativo e possuem conhecimento sobre a ortotanásia, porém esse momento é carregado de dificuldade e sentimentos de angústia, principalmente em relação a aceitação dos familiares. Esse fato pode ser explicado pela falta de abordagem sobre o tema durante a formação e atuação profissional destes profissionais, situação referida por grande parte da amostra estudada. 
Em relação ao perfil dos profissionais de saúde que atuavam no local do estudo, os profissionais da área de enfermagem foram os mais predominantes, bem como a formação em instituições de cunho privado, verificou-se que o fato do profissional possuir ou não especialização em alguma área repercute sobre as escolhas terapêuticas da equipe frente à terminalidade da vida.

Portanto, conclui-se que para que seja alcançado o objetivo de prestação de uma assistência humanizada ao paciente idoso em fase terminal, faz-se necessário a ampliação sobre tal temática. No entanto, recomenda-se a realização de pesquisas futuras mais amplas, que abordem uma quantidade maior de hospitais e profissionais, pois a partir daí será possível traçar estratégias de ampliação do conhecimento acerca dos conceitos aqui discutidos.

\section{Referências}

Almeida, H. R. A., \& de Freitas Melo, C. (2018). Práticas de ortotanásia e cuidados paliativos em pacientes com câncer termin al: uma revisão sistemática da literatura. Enfermería Global, 17(3), 529-574.

Arrieira, I. C. D. O., Thofehrn, M. B., Porto, A. R., Moura, P. M. M., Martins, C. L., \& Jacondino, M. B. (2018). Espiritualidade nos cuidados paliativos: experiência vivida de uma equipe interdisciplinar. Revista da Escola de Enfermagem da USP, 52.

Balla, A., \& Haas, R. E. (2008). Percepção do enfermeiro em relação à ortotanásia. Revista Bioethikos, 2(2), $204-13$.

Bardin, L. (2011). Análise de Conteúdo. Edições 70.

Bifulco, V. A., \& Iochida, L. C. (2009). A formação na graduação dos profissionais de saúde e a educação para o cuidado de pacientes fora de recursos terapêuticos de cura. Revista brasileira de educação médica, 33(1), 92-100.

Borges, M. D. S., \& Mendes, N. (2012). Representações de profissionais de saúde sobre a morte e o processo de morrer. Revista Brasileira de Enfermagem, 65(2), 324-331.

Both, J. E., Leite, M. T., Hildebrandt, L. M., Spies, J., da Silva, L. A. A., \& Beuter, M. (2013). O morrer e a morte de idosos hospitalizados na ótica de profissionais de enfermagem/The dying and death of elderly hospitalized in perspective of nursing professionals1. Ciência, Cuidado e Saúde, 12(3), 558-565.

Cano, C. W. D. A., Silva, A. L. C. D., Barboza, A. F., Bazzo, B. F., Martins, C. P., Iandoli Júnior, D., ... \& Nantes, R. D. S. G. (2020). End of life: conceptual understanding of euthanasia, dysthanasia and orthothanasia. Revista Bioética, 28(2), 376-383.

Cardoso, D. H., Muniz, R. M., Schwartz, E., \& Arrieira, I. C. D. O. (2013). Cuidados paliativos na assistência hospitalar: a vivência de uma equipe multiprofissional. Texto \& Contexto-Enfermagem, 22(4), 1134-1141.

Carvalho Ferreira, A. G., de Miranda Duarte, T. M., da Silva, A. F., \& Bezerra, M. R. (2015). Concepções de espiritualidade e religiosidade e a prática multiprofissional em cuidados paliativos. Revista Kairós: Gerontologia, 18(3), 227-244.

Carvalho, K. K. D., Lerch-Lunardi, V., Silva, P. A. D., Schäfer-Vasques, T. C., \& Coelho-Amestoy, S. (2017). Educational process in palliative care and the thought reform. Investigacion y educacion en Enfermería, 35(1), 17-25.

Creswell, J. W. (2010). Projeto de pesquisa: métodos qualitativo, quantitativo e misto (3a ed.). Artmed.

Felix, Z. C., Costa, S. F. G. D., Alves, A. M. P. D. M., Andrade, C. G. D., Duarte, M. C. S., \& Brito, F. M. D. (2013). Eutanásia, distanásia e ortotanásia: revisão integrativa da literatura. Ciência \& saúde coletiva, 18, 2733-2746.

Flick, U. (2009). Introdução à pesquisa qualitativa. Artmed.

Forte, D. N. F. (Org.). Panorama dos Cuidados Paliativos no Brasil. Academia Nacional de Cuidados Paliativos. São Paulo: Academia Nacional de Cuidados Paliativos, outubro, 2018. 15. https://paliativo.org.br/wp-content/uploads/2018/10/Panorama-dos-Cuidados-Paliativos-no-Brasil-2018.pdf.

Giaretton, D. W. L. (2013). Morte e o morrer: sentimentos dos profissionais da saúde diante do paciente terminal. [Trabalho final de conclusão], Universidade Federal de Santa Catarina/RS, 2013.

Guimarães, R. B., \& Nunes, J. S. S. (2016). Conhecimento do profissional de enfermagem sobre cuidados paliativos em pacientes oncológicos. Revista InterScientia, 4(1), 31-36.

Mendes, J. A., Lustosa, M. A., \& Andrade, M. C. M. (2009). Paciente terminal, família e equipe de saúde. Revista da SBPH, 12(1), 151-173.

Meneguin, S., \& Ribeiro, R. (2016). Dificultades De Cuidadores De Pacientes En Cuidados Paliativos En La Estrategia De Salud De La Familia. Texto \& Contexto-Enfermagem, 25(1).

Moritz, R. D., \& Nassar, S. M. (2004). A atitude dos profissionais de saúde diante da morte. RBTI, 16(1), 14-21.

Nogueira, A. C. C., Oliveira, L. M., \& Pimentel, V. (2006). O Profissional da Saúde e a Finitude Humana. A negação da morte no cotidiano profissional da assistência hospitalar. Textos \& Contextos (Porto Alegre), 5(2), 1-11.

Oneti, C. F., de Oliveira Barreto, D. M., \& Martins, E. L. (2017). Percepção dos profissionais de enfermagem frente à prática da distanásia e ortotanásia. Enfermagem em Foco, 8(2). 
Research, Society and Development, v. 10, n. 1, e18910111619, 2021 (CC BY 4.0) | ISSN 2525-3409 | DOI: http://dx.doi.org/10.33448/rsd-v10i1.11619

OPAS. Fatores de risco para doenças crônicas não transmissíveis nas Américas: Considerações sobre o fortalecimento da capacidade re gulatória. Documento de Referência Técnica REGULA. Washington, DC, 2016. https://iris.paho.org/handle/10665.2/28583.

Santana, J. C. B., Lucas, C. P., Fraga, E. M., Souza, L. A. C., \& Santos, T. M. B. (2014). Ortotanásia: uma visão multidisciplinar acerca do morrer com dignidade. Enferm Rev, 17(1), 14-29.

Santana, J. C. B., Rigueira, A. D. M., \& Dutra, B. S. (2010). Distanásia: reflexões sobre até quando prolongar a vida em uma Unidade de Terapia Intensiva na percepção dos enfermeiros. Revista bioethikos, São Camilo, 4(4), 402-411.

Santos, L. R. G. D., Menezes, M. P., \& Gradvohl, S. M. O. (2013). Conhecimento, envolvimento e sentimentos de concluintes dos cursos de medicina, enfermagem e psicologia sobre ortotanásia. Ciência \& Saúde Coletiva, 18, 2645-2651.

Silva, D. D. J. N., Casimiro, L. G. G., de Oliveira, M. I. S., da Cunha Ferreira, L. B., \& Abelha, F. J. P. A. (2020). A população cirúrgica muito idosa em cuidados intensivos: características clínicas e desfechos. Brazilian Journal of Anesthesiology.

Silva, J. A. C. D., Souza, L. E. A. D., Silva, L. C., \& Teixeira, R. K. C. (2014). Distanásia e ortotanásia: práticas médicas sob a visão de um hospital particular. Revista bioética, 22(2), 358-366.

Silva, L. D. M., de Resende, M. C., \& Andrade, R. Z. (2019). Atitudes de profissionais de saúde em cuidados paliativos sobre a morte e o morrer. Perspectivas em Psicologia, 23(1), 213-235.

Silva, R. S. D., Evangelista, C. L. D. S., Santos, R. D. D., Paixão, G. P. D. N., Marinho, C. L. A., \& Lira, G. G. (2016). Percepción de las enfermeras intensivistas de un hospital regional sobre distanasia, eutanasia y ortotanasia. Revista Bioética, 24(3), 579-589.

Souza, A. M., Pereira, M. H. Q., Teles, B. K. A., de Souza, A. L., dos Santos Pereira, D., \& Pereira, M. L. A. S. (2020). Marcadores de risco cardiovascular em idosos da Estratégia de Saúde da Família/Cardiovascular risk markers in the elderly of a Family Health Strategy. Brazilian Journal of Development, 6(6), 3609436109. 